\title{
The RiSE Climbing Robot: Body and Leg Design
}

\author{
A. Saunders ${ }^{\mathrm{a}}$, D.I. Goldman ${ }^{\mathrm{b}}$, R.J. Full ${ }^{\mathrm{b}}$, M. Buehler ${ }^{\mathrm{a}}$ \\ ${ }^{a}$ Boston Dynamics, 515 Massachusetts Ave, Cambridge, MA, USA 02139 \\ ${ }^{b}$ Department of Integrative Biology, University of California, Berkeley, CA, USA 94720-3140
}

\begin{abstract}
:
The RiSE robot is a biologically inspired, six legged climbing robot, designed for general mobility in scansorial (vertical walls, horizontal ledges, ground level) environments. It exhibits ground reaction forces that are similar to animal climbers and does not rely on suction, magnets or other surface-dependent specializations to achieve adhesion and shear force. We describe RiSE's body and leg design as well as its electromechanical, communications and computational infrastructure. We review design iterations that enable RiSE to climb $90^{\circ}$ carpeted, cork covered and (a growing range of) stucco surfaces in the quasi-static regime.
\end{abstract}

Keywords: climbing robots, bioinspired design, power autonomous locomotion, scansorial agility

\section{INTRODUCTION}

Climbing on artificial surfaces like on the exterior of buildings, or on natural surfaces like trees or cave walls, is desirable for applications like search and rescue, recon, etc., yet challenging to achieve for robotic devices. Practical utility requires that climbing robots must deal with different surfaces and geometries, which precludes the use of special purpose attachment devices like magnets or suction. At the same time, the machines need to be power autonomous, to avoid the hazard and the limitations of a tether. Climbing robots need unprecedented reliability: even one misstep in a thousand can lead to a catastrophic fall.

Previously designed climbing robots have successfully climbed a number of hard, real world terrains. In general however these robots have been designed with a specific subset of terrain geometries or surface types in mind. Surface specific appendages that rely on magnetism [1] and suction [2][3][4] have been implemented in real world applications such as window cleaning and ship hull inspection. Robots like Dante II [5] navigated the complicated terrain in an active volcano using tether assisted climbing and rappelling. More generic climbing platforms such as Lemur [6][7] and the RiSE robots [24] have been making progress towards climbing more generic terrains. The Lemur robot, which can climb near vertical surfaces in a static fashion with prior knowledge of adequate holds, is aiming towards space exploration and service. Mini-

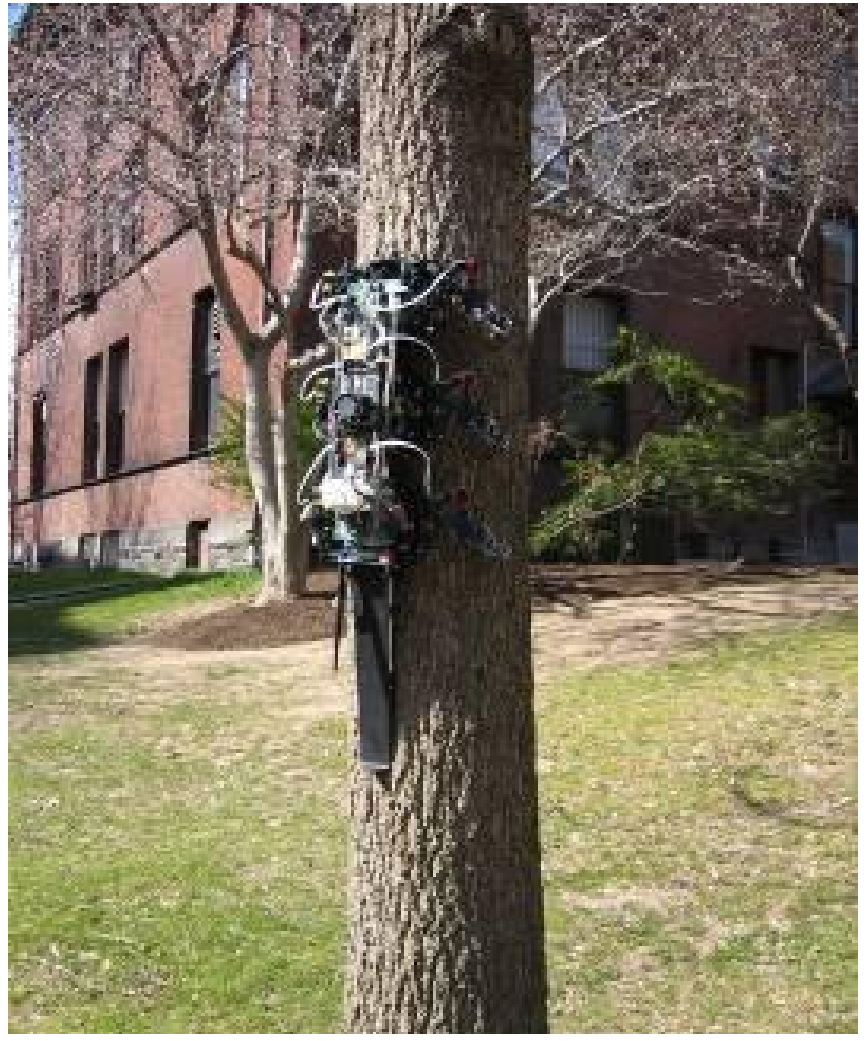

Figure 1: The RiSE robot climbing a tree. Whegs $^{\mathrm{TM}}$ [8], has impressive horizontal mobility [9] and climbs smooth vertical glass surfaces [10]. The sticky-foot based Mini-Whegs ${ }^{\mathrm{TM}}$ and others, like the platforms discussed in [11], show similarities to the gecko inspired climbing work by Full et al [12] on the leg based MechoGecko and the track-based BullGecko. The Mecho-Gecko's three legs are tipped with a pressure-sensitive adhesive, similar in strength to Post-it ${ }^{\circledR}$, and move in a fashion that mimics the

Unmanned Systems Technology VIII, edited by Grant R. Gerhart, Charles M. Shoemaker, Douglas W. Gage, Proc. of SPIE Vol. 6230, 623017, (2006) · 0277-786X/06/\$15 · doi: 10.1117/12.666150 
unroll-and-peel-off manner in which geckos climb. Other biologically inspired designs include snake robots [13][14], where careful design of body posture driven gaits enables them to climb hard surface geometry like pipes and cracks. The ongoing DARPA MDMR program (http://www.darpa.mil/tto/programs/mdmr.htm) investigates concepts using serpentine mobility to achieve new ground robot capabilities for search and rescue applications. Climber III [15], uses a VRAM vortex based mechanism to ascend smooth and uneven flat surfaces such as wall board, brick, and cement. It is also capable of inverted operation on ceilings, but requires human assistance to make the transitions. This platform makes no sustainable physical connection to the wall and the VRAM fans must operate continuously while the robot navigates a vertical wall or ceiling.

RiSE (Robots in Scansorial Environments) is among a new class of vertical climbing robots that make use of biologically inspired design and control to achieve climbing performance and agility that has not previously been attained by robots in "scansorial" environments. The RiSE robot is a step towards integrating the high level of mobility in the horizontal terrain shown by its predecessor, the hexapod RHex platform [16], with the amazing climbing behaviors demonstrated by biological climbers like lizards and insects. The power autonomous RiSE platform (v1.0) was designed to support research into dynamic locomotion in challenging vertical terrain environments, without losing capabilities in level ground operation. The RiSE platform employs biologically inspired attachment dynamics [21] and micro-spine [18] foot structures attached to a highly mobile platform to adhere to complex real world terrain such as trees, brick and other vertical building surfaces such as stucco [19].

RiSE is unique in comparison to many other successful vertical climbing robots since it has the ability to adapt to a large number of surfaces types and geometries. Understanding wall reaction forces, limb trajectories, and surface interactions is key to designing a multifunctional platform capable of climbing difficult terrain without relying on surface specific mechanisms. Careful selection of leg number, leg compliance and foot path generation is required. Climbing hard-smooth surfaces would benefit greatly from integrating advanced materials (gecko-inspired synthetic dry adhesives) into foot designs [19] and fabricating them using rapid prototyping technique like shape deposition manufacturing [20]. In the sequel we discuss the RiSE platform as it currently exists and our approach to these challenges as inspired and informed by our study of gecko, arthropod, and mammalian morphologies and behaviors.

\section{BODY DESIGN AND USE IN ANIMAL CLIMBERS}

A comparative approach to the study of animal climbing could reveal advantages and disadvantage in body and leg structure and function that could inspire the design of a legged climbing robot. Autumn et al. [21] have measured the dynamics of gecko climbing and here we report preliminary results on cockroaches [22].

\subsection{Dynamics of the center of mass in four versus six-legged vertical climbers}

Differences in leg number and placement could lead to different center of mass dynamics during rapid, vertical climbing. Surprisingly, common center of mass dynamics are generated by animals that differ in leg number. The dynamics of the center of mass measured in four-legged climbing geckos [21] and six-legged climbing cockroaches are remarkably similar. Both use a trotting gait during rapid climbing resulting in a different number of legs in contact with the surface. Two legs of the gecko and three legs of the cockroach sum to act as a single leg accelerating the center of mass upward cyclically. Both animals effectively place their feet during rapid climbing, so that minimal fore-aft deceleration forces are generated by legs, thus reducing the power requirements necessary to ascend. As they accelerate upward in the fore-aft direction, these sets of legs pull the animals laterally. Geckos attain these lateral dynamics by pulling toward the side of the body on which the fore leg is in contact with the surface, because it exceeds the pull of the hind leg [21]. The resultant force of the tripod in the cockroach pulls the animal toward the side of the body on which the middle leg is in contact with the surface. Differences in leg number do not reveal any advantages with respect to center of mass dynamics during rapid vertical climbing on surfaces with effective footholds, but do suggest a common pattern worthy of consideration in robot design. 


\subsection{Single leg function in vertical climbing}

Autumn et al., [21] discovered that geckos produce acceleratory fore-aft forces during rapid climbing with both fore and hind legs. In the lateral direction, all legs pull towards the mid-line of the body. Fore legs pull the head toward the vertical surface, while hind legs push the body away. Normal wall forces are small, but were sufficient to counter pitchback. No measurable attachment or detachment forces occur at the beginning or end of a step.

Although geckos and cockroaches differ in leg number and placement, single leg forces show surprising similarities during rapid climbing. Cockroaches generate fore-aft accelerating forces with all three legs of the tripod [22]. Cockroaches pull toward the mid-line as do geckos, but not with all legs. The middle leg of cockroaches functions more like a hind leg in geckos, while the hind leg in cockroaches generates near zero lateral force. Like the geckos, the cockroaches balance pitchback moments due to gravity by using the fore leg normal forces to pull the head toward the surface. Hind legs push away from the surface. Middle legs in cockroaches generate small normal forces.

To better understand the origin of the similarities, we developed a dynamic model of insect climbing. The model had the advantage of manipulating the effectiveness of the footholds. We hypothesized that differences in body and leg design may become more apparent during the resulting perturbations.

\subsection{Biological inspiration for a climbing robot}

\subsubsection{Simple dynamic model of a climbing robot}

A dynamic model of insect climbing can reproduce both the dynamics of the center of mass and the forces developed by individual legs (Figure 2(a,c)). During the model's development, we were able to parameterize key aspects of leg function. Preliminary exploration of parameter variation continues to give us insight into the animal's leg configuration, To determine the effect of leg number and compliance on vertical climbing in robots, we developed two planar models that captured the general features of RiSE (Figure 2(b,d) Figure 2(a,b)) The leg morphology of the models was similar to that of the robot. Compliance, damping, as well as foot adhesion could be varied. Parameters were adjusted so that the simulation had compliances comparable to those on the robot and developed forces qualitatively similar to those used by geckos [21] and cockroaches [22]; Figure $3(\mathrm{c}-\mathrm{f})$. The motors that drove the 4-bar linkage maintained a fixed angular velocity. Foot contact was established during fixed phase of the cycle such that a rigid pin joint was established with the background during touchdown. For a range of parameters, the models stably ascended the wall at approximately $15 \mathrm{~cm} / \mathrm{sec}$.

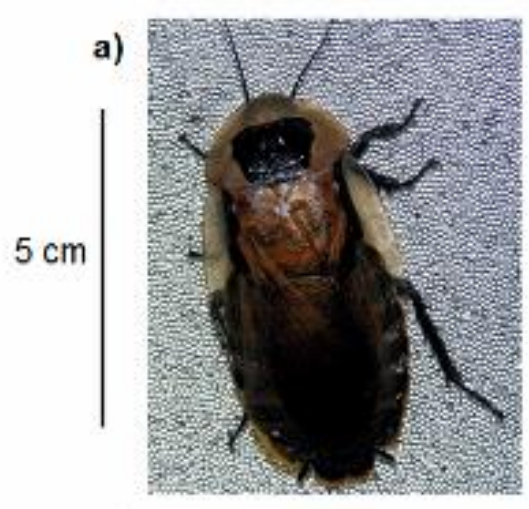

c)

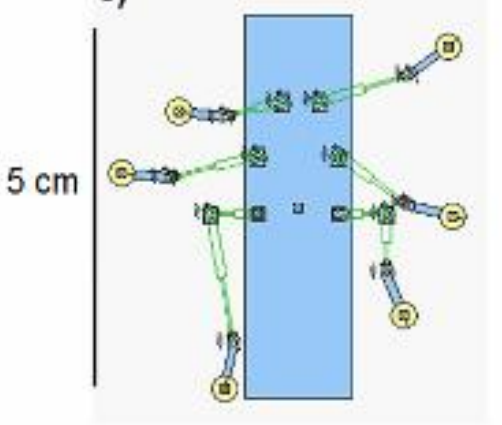

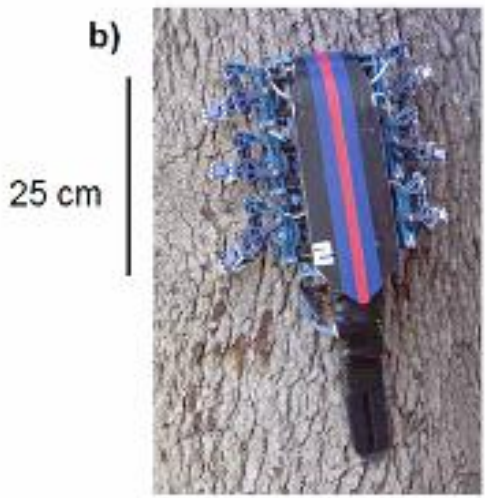

d)

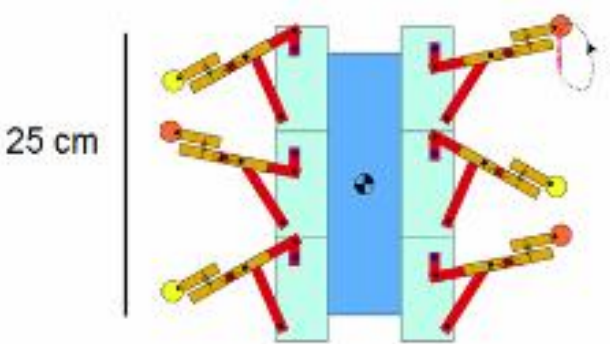

Figure 2: Climbing insect (a), robot (b), insect model (c) and robot model (d). The numerical models generate similar wall reaction forces measured in the animals and robots and can be used to investigate leg configuration, trajectory and compliances. 


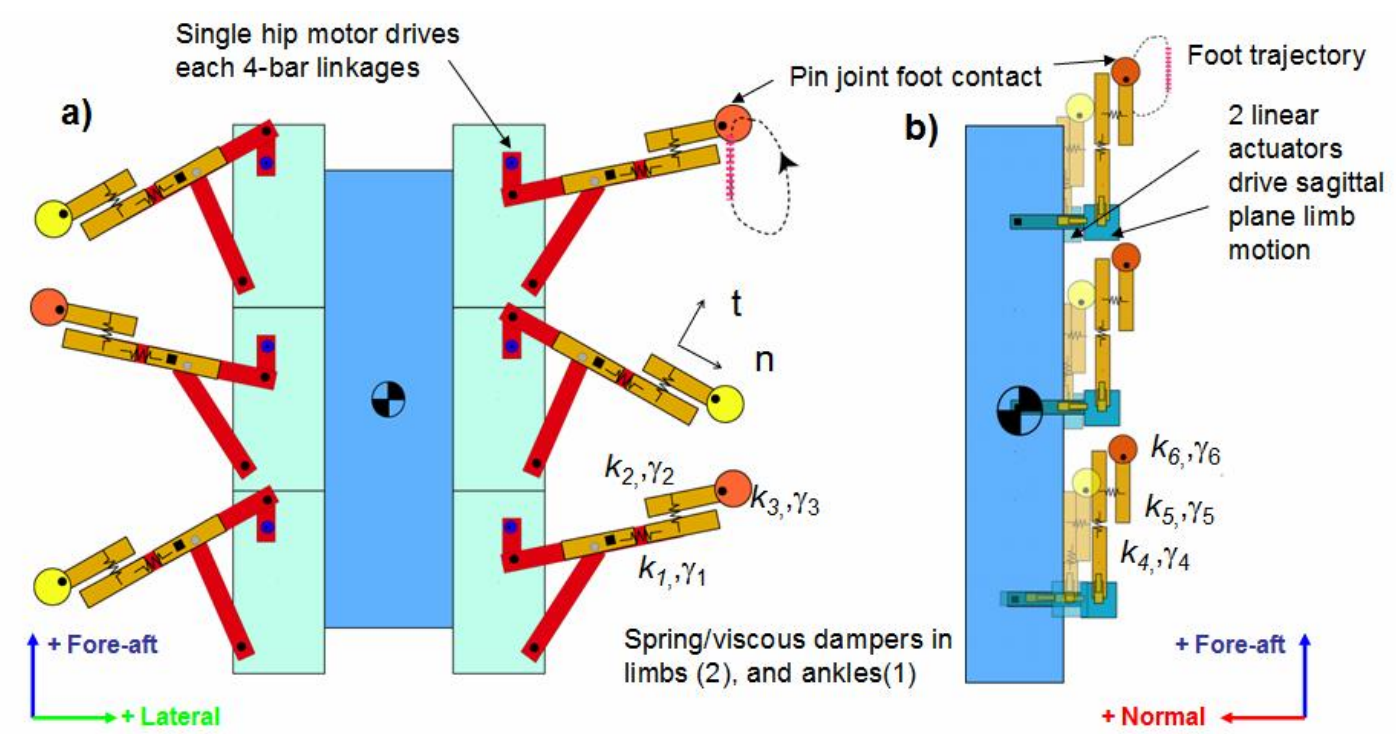

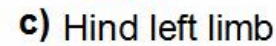

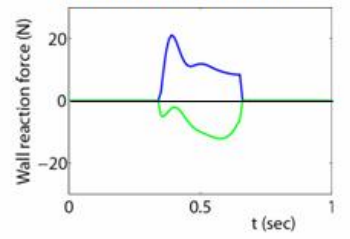

d) Fore right limb

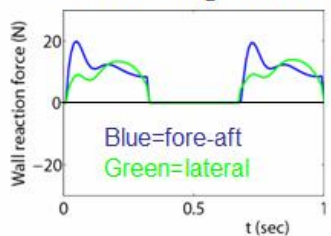

e) Hind limb

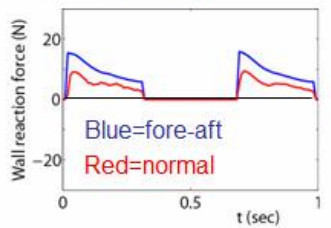

f) Fore limb

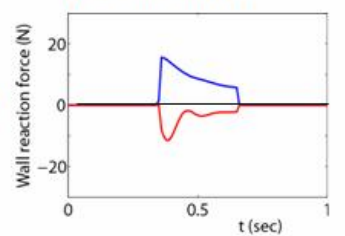

Figure 3: Two RiSE robot simulations in the a) frontal and b) sagittal planes with corresponding wall reaction forces c-f.

Robot parameters for the simulation in Figure 3(a), the frontal plane model include body mass $=2 \mathrm{~kg}$, motor frequency $=1.5 \mathrm{~Hz}, \mathrm{k}_{1}=2000 \mathrm{~N} / \mathrm{m}, \gamma_{1}=30 \mathrm{~N}-\mathrm{sec} / \mathrm{m}, \mathrm{k}_{2}=5000 \mathrm{~N} / \mathrm{m}, \gamma_{2}=50 \mathrm{~N}-\mathrm{sec} / \mathrm{m}, \mathrm{k}_{3}=0.001 \mathrm{~N}-\mathrm{m} /$ degree, $\gamma_{3}=0.0001 \mathrm{~N}-$ $\mathrm{m}$-sec/degree. Robot parameters for the simulation in Figure 3(b), the sagittal plane model body mass $2 \mathrm{~kg}$, motor frequency $=1.5 \mathrm{~Hz}, \mathrm{k}_{4}=2000 \mathrm{~N} / \mathrm{m}, \gamma_{4}=100 \mathrm{~N}-\mathrm{sec} / \mathrm{m}, \mathrm{k}_{5}=5000 \mathrm{~N} / \mathrm{m}, \gamma_{5}=100 \mathrm{~N}-\mathrm{sec} / \mathrm{m}, \mathrm{k}_{6}=0.1 \mathrm{~N}-\mathrm{m} /$ degree, $\gamma_{6}=0.0001 \mathrm{~N}-$ $\mathrm{m}$-sec/degree. Each foot has a mass of $0.1 \mathrm{~kg}$. Foot wall reaction forces generated by the model are inspired by forces measured in climbing cockroaches and geckos. c,d) Wall reaction forces in the fore-aft (blue) and lateral directions for fore and hind limb. The sign convention is such that positive wall reaction forces point up, counter to gravity (fore-aft), right (lateral), and out of the wall (normal). Both limbs accelerate the robot up the wall while lateral forces pull in toward the mid-line. e,f) Wall reaction forces in the fore-aft (blue) and normal directions for fore and hind limbs. Both limbs accelerate the robot up the wall. Fore-limbs pull the front of the robot to the wall while hind limbs push the rear away from the wall. The normal forces serve to counter overturning moments generated by the action of gravity on the center of mass. The models are numerically integrated in the Working Model 2D environment. The vectors indicate direction of wall reaction forces in plots c-f.

\subsubsection{Leg number and stability}

To examine stability when legs cannot develop effective ground reaction forces, we degraded foot-wall adhesion for certain legs. These legs could not develop normal or shear forces with the wall. In the other legs, adhesion could be developed provided the torque in the ankles did not exceed a critical value. We found that for both six-legged models, degrading the contact in any two legs did not affect stability or performance of the climber significantly. In the frontal plane model, yaw increased slightly, and speed decreased slightly. In the sagittal plane model, pitching oscillation amplitude increased during a stride. When contact was degraded in three legs, the frontal plane model developed catastrophic yaw and was unable to ascend even a single body length. The situation was even more dramatic in the sagittal plane model. Removal of contact for three legs resulted in immediate catastrophic pitchback. Our results indicate that climbing with only four legs imposes strong constraints on the reliability of the adhesive mechanism, and requires major alteration in foot-force pattern to gain stability upon perturbation. Climbing in four-legged animals using 
a trotting gait (two limbs on the wall during stance) is challenging, because perturbation of a single leg requires the only remaining leg to support the weight on the climber, resulting in large leg stresses. The situation in the sagittal plane is worse, because the legs are operating against each other in the normal direction (Fig. 3e,f). Since hind legs typically operate to push the center of mass away from the wall (Fig. 3e), the loss of fore-leg contact results in the absence of normal adhesive to counter pitchback. Thus, the hind-leg must change its force generation to counter pitchback or perhaps use its tail. The loss of a fore-leg contact in a six-legged climber is not as catastrophic, because the middle leg can still provide normal adhesion, as well as fore-aft support of weight.

\subsubsection{Importance of tuned leg and ankle compliance}

Varying the model's leg and ankle spring stiffness had significant effects on performance. We found that leg springs with similar values were needed in both models. Increasing axial compliance $\mathrm{k}_{1}$ in the frontal plane model resulted in increased climbing performance (decreased yaw) until the forces developed in the foot constraint became too large. We found that rigid ankles resulted in large foot forces and did not allow stable climbing. Flexion in the ankle was necessary to allow the model to ascend. The ankle spring constant in the frontal plane $\left(\mathrm{k}_{3}\right)$ was $1 / 100$ that in the sagittal plane simulation $\left(\mathrm{k}_{6}\right)$. High ankle compliance in the frontal plane appears critical to prevent large wall reaction forces. Low stiffness is not advantageous in the sagittal plane because it allows larger rotational displacements. We conclude that compliance must be adjusted to develop effective force patterns over appropriate durations. As in models of locomotion for level running [23], inappropriate choice of compliances results in large oscillations in yaw and pitch, and can lead to instability. However, there are stronger constraints in climbing, because wall reaction forces must not exceed the strength of the adhesion mechanism.

\section{ENGINEERED BODIES FOR CLIMBING}

The initial engineering effort for the RISE robot focused primarily on the design and implementation of passive, compliant appendages, and their integration into a power- and computation- autonomous robotic platform. Longer term emphasis will be on linking carefully designed mechanical structures with sensor-based strategies for adjusting stance and motion parameters, recovering from slips when traction is marginal, and nimbly negotiating scansorial terrain in a dynamical manner. We have taken principles from biological climbers to design the legs and body of RiSE. We used discoveries on the effect of leg number, trajectory and attachment mechanisms that can allow rapid climbing dynamics with high levels of agility. The following covers how this inspiration was translated into the RiSE platform design.

\subsection{Review: Initial Design of RiSE 0.1}

The initial prototype of the RiSE (v0.1) platform [24] was completed and tested in 2004. Each of the six legs is powered by two small electric motors through a differential gear mechanism. The leg mechanisms are under-actuated planar mechanisms approximately co-planar to simple curved climbing surfaces. The platform currently weights just over $3 \mathrm{~kg}$ and has a payload capacity of nearly $1.5 \mathrm{~kg}$. Three onboard batteries, powering control and motor circuitry, provide runtimes in excess of one hour.

Onboard electronics as shown in the system block diagram of Figure 4 control leg motion, manages communications, and services a variety of sensors. The platform is computationally autonomous; communication to the operators control unit (OCU) is achieved through a wireless 802.11 link, allowing the robot to be controlled remotely. Due to the small size of the RiSE platform, a custom electronics suite was designed to integrate a small form factor PC, memory, and a solid state IMU. On platform communication and control is achieved through a custom, serial based, distributed communications protocol referred to as RiSEBus. RiSEBus is used to connect sensors and motor control boards to the CPU module. The sensors include joint position sensors for each leg, leg strain sensors and foot contact sensors. Due to the specific form factor requirements, weight budget, and the low inductance of the high performance coreless DC motors, no COTS electronics could be found to satisfy the requirements of the RiSE platform. As a result each pair of hips is controlled via a high frequency custom motor amplifier board.

The hexapod design chosen for the RiSE platform was informed by the simulation of the robot as inspired by cockroach and gecko climbing. As reported the simulation was capable of climbing with only two legs, suggesting a quadruped design was adequate for climbing. However, the simulation also showed than any foot failures resulting in less than two feet in contact with the surface resulted in catastrophic failure. When combined with the desire to maintain mobility on 
the level, we chose a more conservative, redundant hexapod configuration. Experiments with the v0.1 platform supports the simulation resuts: catastrophic failures (complete detachment from the wall) did not occur until less than two feet were attached to the wall.

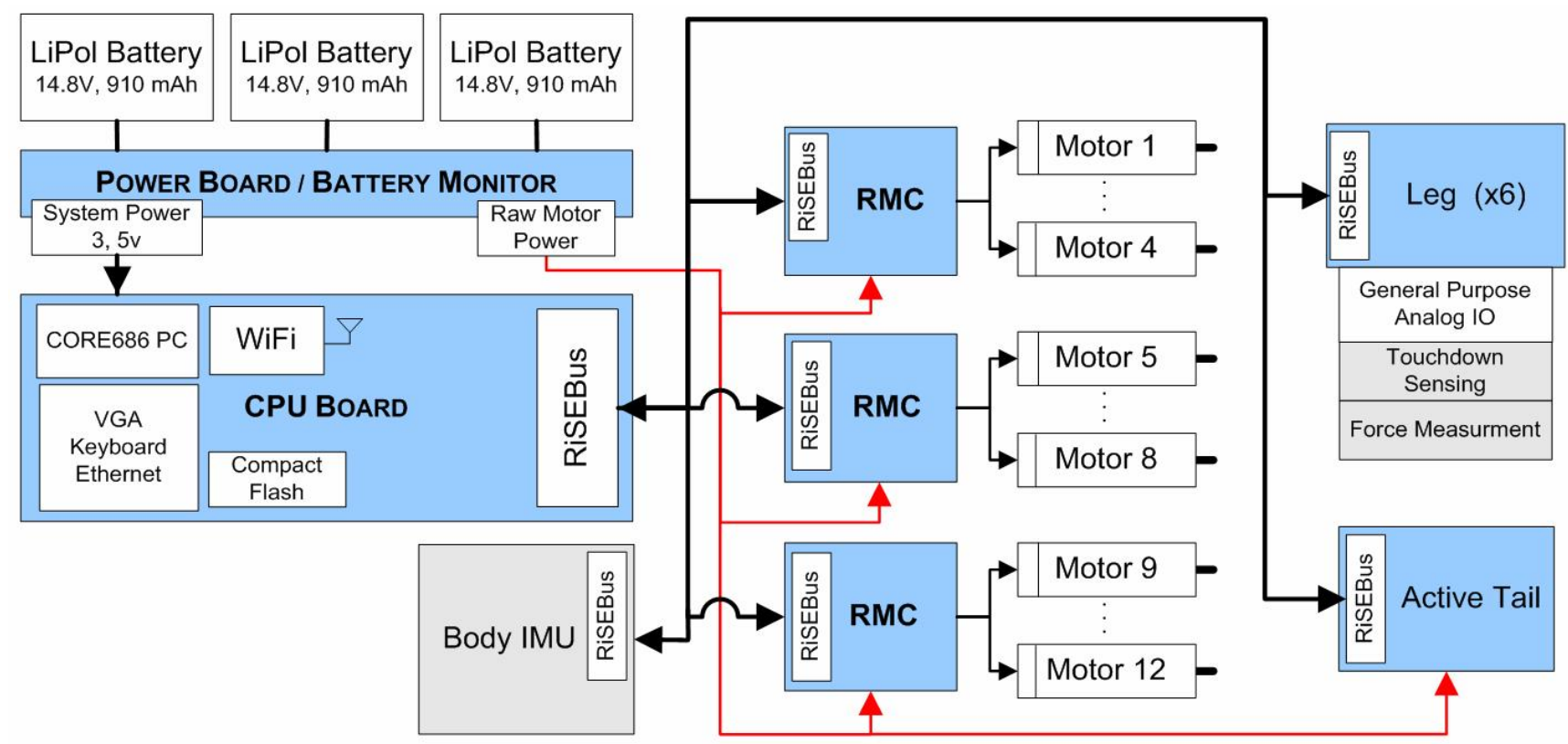

Figure 4: System block diagram of the RiSE robot.

The choice of a hexapod configuration forced careful design of limbs since weight budgets permitted only two actuators per leg. As a result the key to locomotion in both the vertical and horizontal terrain with the same underactuated leg mechanism was to exploit a carefully designed foot path (generated by a four bar planar leg) and correctly tuned compliance as suggested by biology. Figure 6(a) shows the top view path of a foot, co-planar to the climbing surface, attached to the four bar linkage. The free toe trajectory (solid line) is modified based on the touchdown and liftoff points to engage the leg compliance with the climbing surface, via the independent abduction/adduction or "wing" degree of freedom (Figure 6(b)). The constrained toe trajectory (dotted line) exploits the compliance to apply the correct "pull in forces", similar to how geckos and other animals climb, as shown in Section 2. During locomotion on horizontal terrain, the crank direction of the four-bar is reversed, and the leg compliance provides a compression (left portion) section during stance. Because there is no direct control over leg axis force during stance careful selection of leg axis compliance was critical. Simulations presented in Section 2 showed that the correct compliance was important to invoke the natural body dynamics and foot force observed in biology.

Attached to the planar linkages of the four-bar is a compliant structure between the rigid upper leg to the lower leg. Early designs, Figure 5 (b), used spring metal flexures to achieve partially decoupled adjustment of lateral and normal leg compliance. Experimental results suggested increasing the normal compliance allowed for more control of normal leg forces, critical for a platform designed to handle a wide array of materials, each with their own compliance. Ultimately a mechanism with no initial normal compliance proved to be the most robust solution. As a result a mechanism, shown in Figure 5 (b), was designed to address the need for different leg axis compliance in the running (compressive) and climbing (tension) loads. The antagonistic spring configuration however limited differences in spring constants and pre-load values. Simulation, supported by empirical results, suggests independent adjustment of spring constant and pre-load, as well as the addition of damping, for the leg compliances was critical for tuning efficient scansorial locomotion over a wide variety of surfaces. Additionally the reliability and repeatability of the crossed extension spring design in

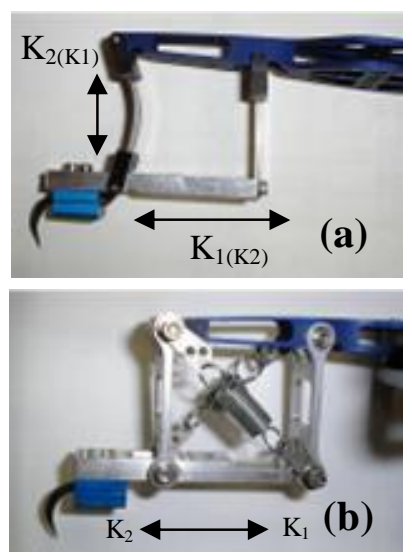

Figure 5: Early versions of the compliant legs used on RiSE v0.1 
Figure 5(b) was low so the leg mechanism was redesigned for the v1.0 version of the platform and is reported in the following section.
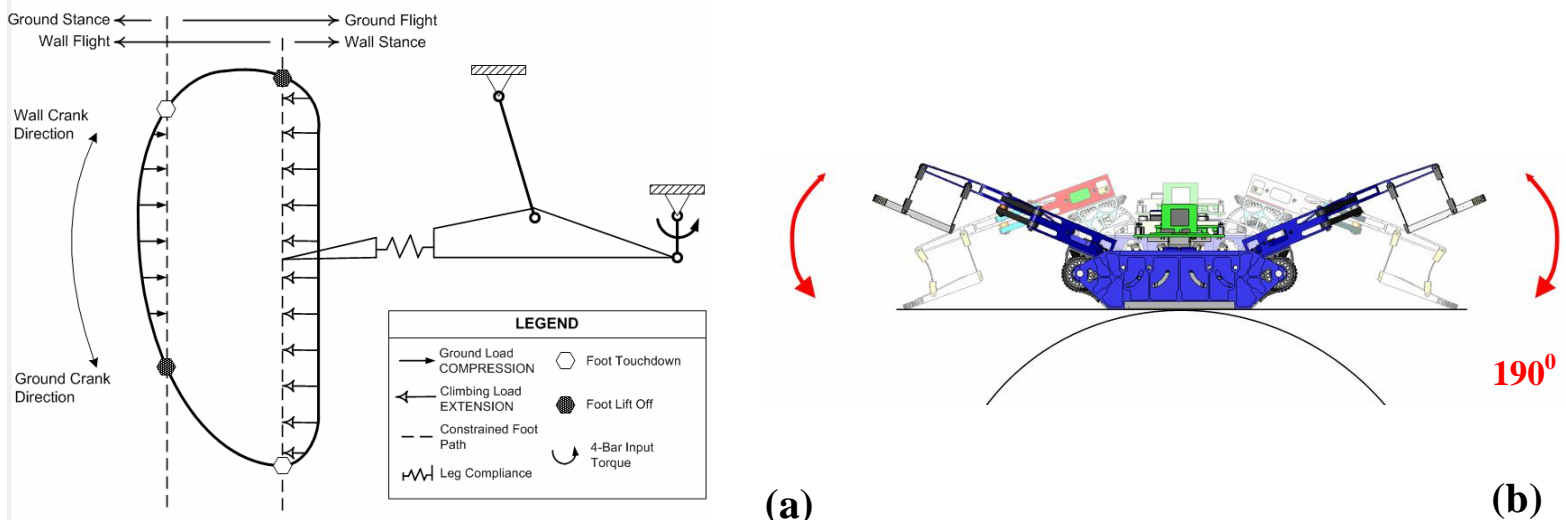

(a)

(b)

Figure 6: The kinematics of the RiSE leg. (a) Foot trajectory produced by the four-bar leg mechanism. (b) Range of motion of the "wing" degree of freedom.

The twelve actuators on the RISE platform are Maxon [25] brushed DC servo motors, selected for their high power to mass ratio and adequate selection of small frame size, fractional HP motors. Other manufactures, such as Faulhaber, produce similar high performance fractional DC servo motors. Brushless motors were not chosen because they require more complex, larger, and heavier drive circuitry. Maxon uses a light weight coreless motor design, combined with rare earth magnets, to deliver high current with low mass of the system. The disadvantage of using a coreless motor, in addition to their cost, is a low thermal mass (current + time $=$ heat). Careful heat dissipation and current limiting is essential for demanding dynamic applications that benefit from overdriving the motors. With small coreless motors it can be tricky to use direct motor case temperature feedback as the low thermal mass of the windings compared to the motor case can lead to burnout before the case temperature changes significantly. A multistage current limiting scheme was used to protect the motors on the RiSE platform. Commanded current is allowed to pass the maximum continuous current as specified in the data sheet for a specified period of time. This time was experimentally determined by increasing motor over current and watching the steady temperature of the motor. The final stage forces the safe current for a period of time adequate to allow the motor temperature to recover. Determining how much a motor can be overdriven requires many experiments, often resulting in damaged motors. Using methods like this allows control to exploit the full potential motor performance, important in mass critical dynamic application.

For the RiSE platform, twelve motors are used to drive six limbs. The motors were sized for a $2 \mathrm{~kg}$ body mass and a climbing velocity of $0.25 \mathrm{~m} / \mathrm{s}$ using at least two legs, which is four motors on the RiSE platforms, after which RiSE looses attachment to the wall. The total drive train efficiency $\eta$ used was a conservative $50 \%$ based on prior experience building small robots. The relationship

$$
P_{m}=\frac{m g v}{\eta N_{m}}
$$

was used to determine an estimate of motor power capabilities for vertical climbing, where $m$ is the mass of the robot, $g$ is the gravitational constant, $v$ is the robot speed, and $N_{m}$ is the number of motors that will share the climbing load.

The resultant power requirement per motor was $2.5 \mathrm{~W}$, which made the Maxon RE13 series, and up, potential choices. If we were only concerned about the net energy needed to climb at a particular velocity the above logic can be used reach a good estimate for motor power requirements. In reality, during each stance phase, the robot will be accelerating and decelerating due to inefficient foot placement. Autumn et.al. [21] measured the effective acceleration against gravity for vertically climbing geckos. On level ground a constant average velocity means the acceleratory forces must sum to equal the declaratory forces. Climbing geckos generate total mechanical energy that is at most $15 \%$ greater than 
the change in potential energy compared to an ideal model. To account for this effect, we multiply the right hand side in (1) with a constant velocity factor of 1.15.

The resulting total mechanical power is $2.8 \mathrm{~W}$, near the limits of the RE13. Because robots rarely achieve the efficiency of their biological counterparts the decision to jump up to the next frame size was made. The RE16 provides $4.5 \mathrm{~W}$, a safe margin for our design. In the end the target weight of $2 \mathrm{~kg}$ was exceeded by almost $1 \mathrm{~kg}$. With this increased mass, the required climbing power would be $4.2 \mathrm{~W}$, close to the motors peak capability. Additionally, significant power is required to swing the flight legs into position to start stance, therefore the $4.5 \mathrm{~W}$ Maxon motor was chosen. It is however worth noting that we are currently limited by how fast we can swing the flight leg and re-attach (velocity limited) rather than torque limited, a result of gearing towards strength and not speed.

Once the motors were selected, planetary gearing was used to fine tune the strength vs speed for our particular velocity mass combination. For this the gear ratios of additional drive train components, such as the differential hip mechanism, as well as leg kinematics needs to be taken into account. It is worth noting, in the case of Maxon Motors, that gearbox selection does become more challenging as motor size decreases, forcing the burden on external gearing which can often be costly in terms of weight, size and efficiency when compared to a planetary gearbox.

A unique, differential based, hip mechanism allowed for body mounted actuators to drive the two DOF leg mechanisms while minimizing leg mass (to less than $2.5 \%$ of the body mass). As a result the distal leg mass is low, reducing foot impact and inertial effects while actuating both DOF. The combined motions of the two actuators can control the two DOFs of the leg either independently or simultaneously. When both motors in the differential are driven purely in the same directions at the same speed the wing, or abduction/adduction, DOF is actuated. When the motors drive purely in opposite directions at the same speed the crank portion of the four-bar mechanism is actuated, causing the foot to travel in the path depicted in Figure 6(a). And finally, any other combination of directions and speeds actuate both DOF simultaneously.

\subsection{Iteration: Design Critique and Revision for RiSE v1.0}

The first RiSE platform, as discussed above, was designed to operate primarily in the quasi-static regime. As a result the design focused on proving the mechanical concept in a fashion that erred on the side of "strong enough" not "fast enough". During the redesign several key issues were addressed: reduction of the CG offset from the wall, increase in drive train efficiency, improved compliant leg mechanisms, and modularization of the body.
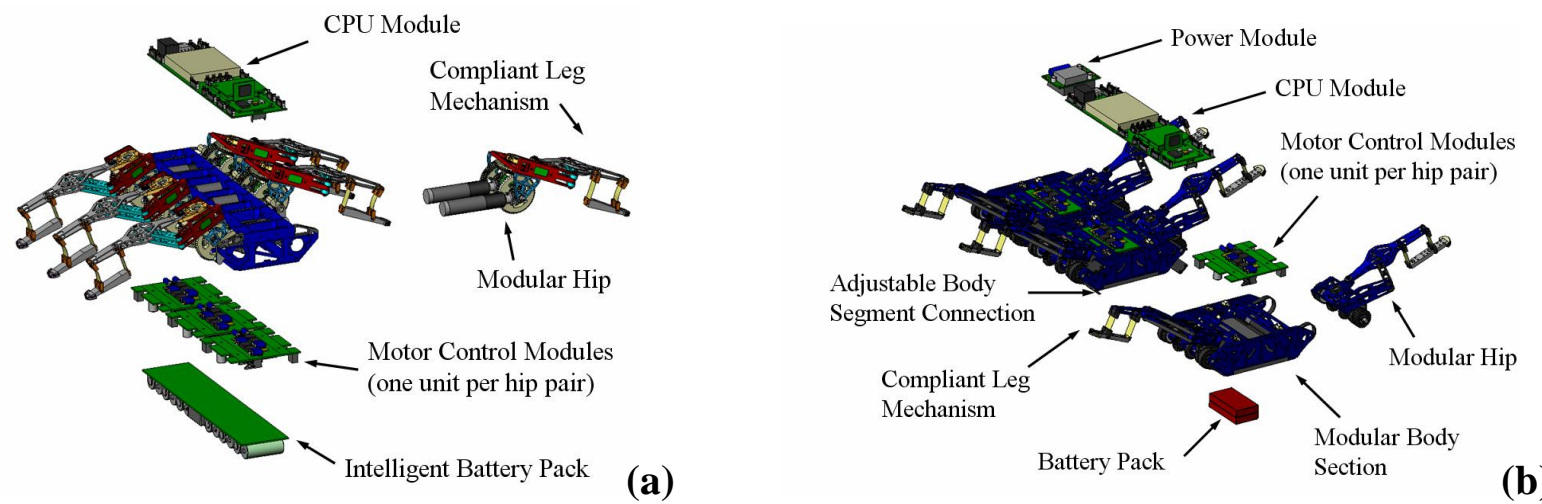

Figure 7: Layout of the v0.1 (a) and v1.0 (b) platforms. Note the v1.0 revision includes modular body sections.

The most significant change in the v1.0 platform was the separation of the body into three identical body modules, one for each leg pair. This modular design allows for easy adjustment of fore/aft foot spacing as well as enables future work on flexible body sections. A side by side comparison of the v0.1 and v1.0 platform is depicted in Figure 7. The body module was redesigned to move the motors as close to the wall as possible. Reordering the stacking of components resulted and switching to different battery technology resulted in a $2 \mathrm{~cm}$ decrease in CG offset from the wall during normal climbing posture. This CG change is illustrated in Figure 6(b) where the v1.0 platform is shown over v0.1. The 
reduction in CG offset significantly reduces the pitch-back moments about the rear attachment points. Moving from a large NMh battery pack to much more compact LiPol packs allowed for the battery to be integrated internal to the module, opposed to the base mounted scheme on v0.1 of the platform. This upgrade in battery chemistry was made possible by the recent advances in LiPol technology, heavily driven by the demanding high current RC industry. The $910 \mathrm{mAh}, 14.8 \mathrm{~V}$, LiPol batteries used on the RiSE platform are capable of discharging at $15 \mathrm{C}$ (14 A) continuously

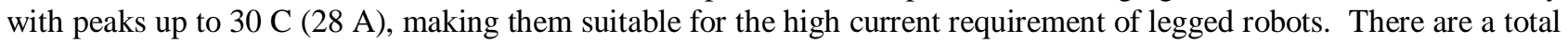
of three $14.8 \mathrm{~V}$ battery packs (one in each module) connected in parallel, enabling 15, 30 and 45 minutes runtime configurations.

Large improvements in performance were realized by improving the efficiency of the drive train. The intermediate connection between the output of the Maxon planetary gearbox and differential, previously unreliable bevel gear set, was replaced with Gates PowerGrip ${ }^{\circledR}$ GT $^{\circledR}$ drive belts. Additionally the tolerances in the mechanical differential structure were increased. The result was a more efficient drive train capable of transmitting higher forces without damage. Experimental results showed significant limits in joint velocity and excessive strength, therefore the gear ratio was decreased to increase leg speed for the v1.0 platform. The belt style drive enabled a tunable drive via interchangeable DriveN and DriveR pulleys. For the v1.0 platform the intermediate gear ratio was changed from 1:1.5 on v0.1 to 1:1 on v1.0. The result was a $50 \%$ increase in climbing speed. When combined with improved speed scaled control schemes, as discussed by Rizzi et. al. [26], the total gain in climbing speed was $80 \%$ from the v0.1 platform. Additionally, the mean time between drive train servicing went from days to months.

Newly redesigned compliant legs, as depicted in Figure 8, make use of standard RC truck shocks to achieve independent control over spring constant, preload and damping. As behaviors approach the dynamical regime, leg compliance is critical as demonstrated earlier by simulations used for tuning the robot. The simulation shown in Figure 3 suggested that inappropriate selection of compliance and damping could lead to large oscillations in yaw and pitch. More importantly for climbing, even in the quasi-static regime, is that wall reaction forces do not exceed the strength of adhesion mechanism. Appropriate compliance can buffer the foot loads appropriately as body moves up the wall. The shocks have built in oil damping adjustable through six standard oil weights and three piston bypass hole sizes. Additionally spring kits, with an assortment of seven spring constants, allow for quick tuning of shock compliance. The kinematics structure allows both shocks to be adjusted independently with no coupling, alleviating the challenges faced when tuning the previously antagonistic spring pairs. It is worth noting that the addition of these shocks increased the mass of the leg substantially ( $150 \%$ increase with respect to the initial leg mass). This additional mass was determined acceptable given the gained flexibility in determining the correct leg compliances for scansorial locomotion. Ultimately, this adjustable mechanism will be replaced by weight optimized design.
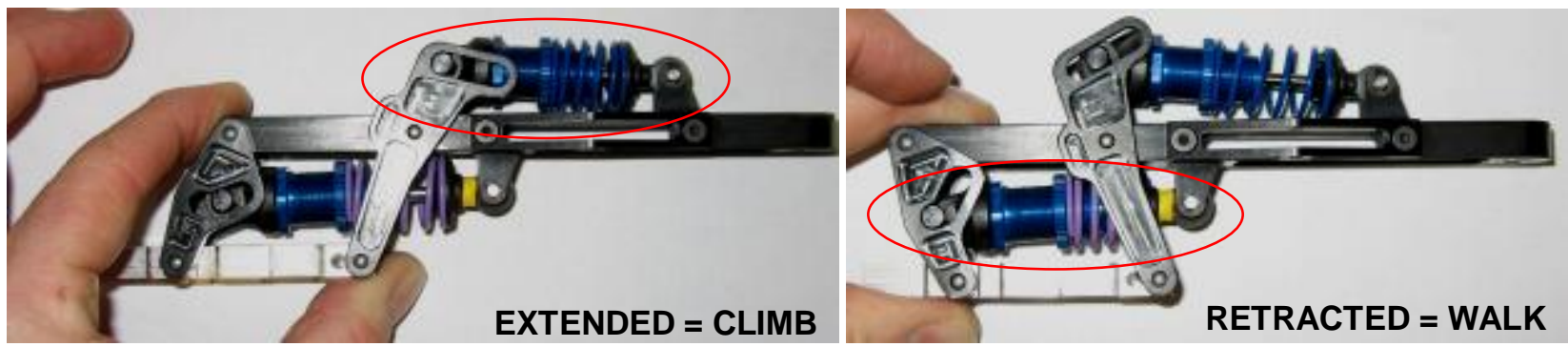

Figure 8: The new compliant leg structure designed to enable independent tuning of extension and compression compliance as well as damping.

As terrain becomes more complicated simple open loop climbing gaits start to fail. As a result, the v1.0 platform needed good tactile sensors to enable more complex feedback climbing behaviors. As a result several new sensors were added to the v1.0 platform, providing 3 axis force measurement each foot. The leg axis force measurement uses spring constants and a Hall Effect based position measurement of the swing link structure. For the other two axes, normal and traction, the v1.0 lower leg structure was converted into a two axis force cell. The load structure shown in Figure 9 was machined from a single piece of 7075-T6 aluminum. The normal force sensing element is a single bending beam, shown under stress in Figure 9(b). The traction force sensing element is a four bar structure machined around the 
normal force beam, shown in Figure 9(c). Both elements are instrumented with a full, temperature compensated, strain gauge bridge. The strain gauge signals are conditioned by a small leg mounted signal conditioning node that connects to the PC via RISEBus. The load cell structure provides linear outputs and shows no discernable coupling between the traction and normal axis. The two pins (red, blue) shown in Figure 9 (a) are connected to the upper leg via parallel swing links, as shown in Figure 8. Four bolt holes on the distal end of the lower leg structure provide general purpose foot mounting. The red pin is fixed from translating to the normal force beam, this means that all traction loads applied to the foot result in a parallel displacement with respect to the read and blue swing link pins. Overload protection for the normal force direction is provided by a slightly larger counter bore in the outer walls, depicted as a small red circle around the red pin. Overload for the traction force direction is provided by the normal bending beam making contact with the outer wall.

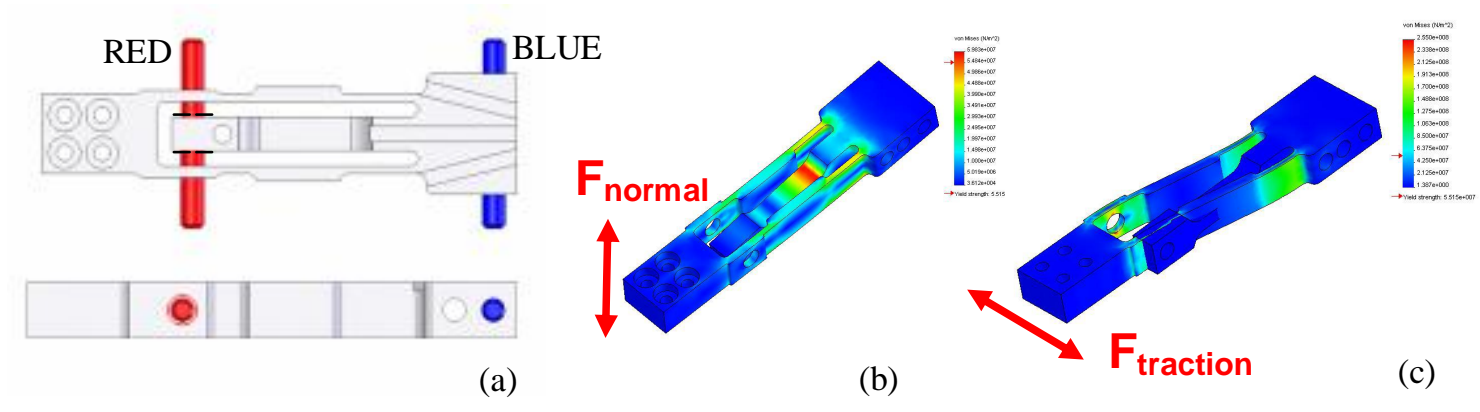

Figure 9: The lower leg structure (a) forms a two degree of freedom force sensor. Normal (b) and traction (c) forces create stress concentrations used to excite a strain gauge bridge.

We currently use different feet types for different surfaces, as shown in Fig. 10. Cutkosky et.al.[19] describes foot development for the RiSE platform, including new micro-spin feet for climbing hard-rough surfaces and the ongoing development of biologically inspired adhesive foot structures. The four feet types shown in Figure 10 include a single point dactyl foot, a spherical rubber walking foot, a multi contact spiny foot [18][27] and finally a lamellar structured sticky foot. As the project moves forward these feet will merge together into one multi function foot capable of handling all regimes of walking and climbing. For now the dactyl foot is used to climb carpet, trees, chain link, wood planks, and in the preliminary brick gaits. The walking foot is used for all horizontal mobility tests. The spiny foot us being used to climb vertical stucco. And finally the lamellar structured sticky foot is used on hard smooth surfaces such as acrylic and glass. The spiny foot represents the clearest path to the future. The first integrated foot, under development by Cutkosky et.al., integrates the spiny and sticky foot technologies and will eventually host the synthetic adhesive under development by Fearing et.al.[28]. It is worth noting that each of these feet function in a way that is not purely surface specific. For example the dactyl functions on smooth-soft surfaces, such as carpet, through hard rough surfaces like tree bark. The spiny foot covers some of the functionality of the dactyl but enables attachment to much more difficult hard surfaces where surface penetration is not possible. The sticky foot works well up to a certain climbing angle on hard smooth surfaces such as glass, metal and smooth concrete. In the future one highly capable foot will function equally well on all surfaces from smooth-hard to soft-rough.

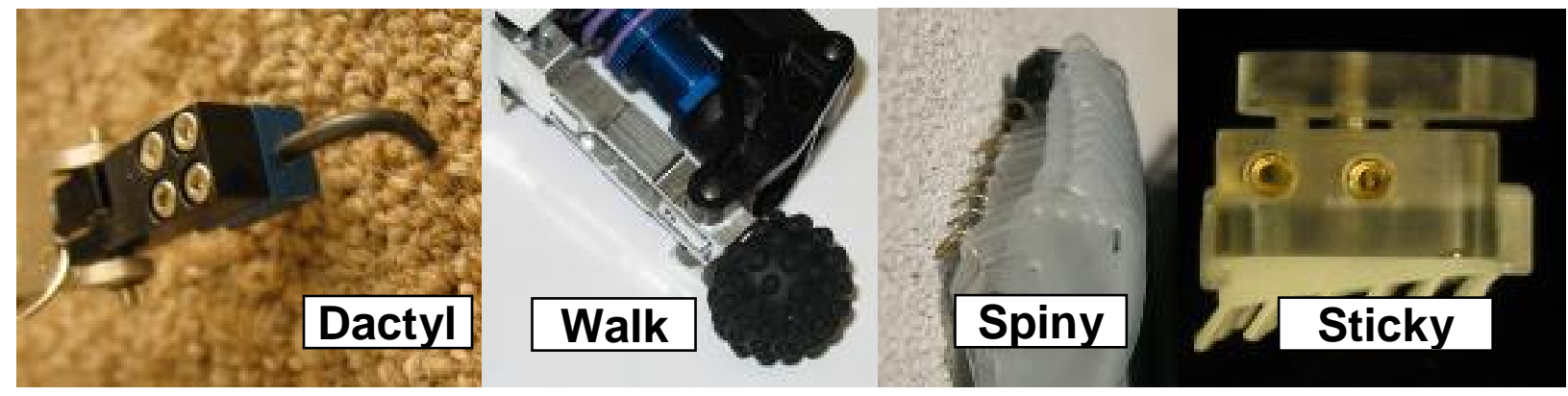

Figure 10: Photographs of four different types of feet used on the RiSE platform. 


\section{RESULTS: STRENGTH, ENDURANCE AND SPEED TRIALS}

To validate the performance of the RiSE platform, independent testing was completed at the Southwest Research Institute (SwRI); the results of the tests are reported in Figure 11. The current testing covers the following regimes for vertical locomotion: Soft-Rough, Soft-Smooth, Hard-Rough, and Hard-Smooth. Initial testing was limited to simplified surfaces that are easy to duplicate in a testing facility. Additionally, tests for drop and crush protection, payload capabilities and horizontal mobility were completed. Speed values are presented primarily for the v0.1 platform with preliminary results for the new v1.0 platform where available. All climbing and walking requires careful behavior development [26], with a variety of gaits and transitions crafted to handle climbing and maneuvering on a wide number of scansorial surface types and geometries. Rizzi et al. [26] provides an in-depth look at the control methods for the RiSE platform. In the following paragraphs several surface types are discussed.

The majority of control and foot development has focused on climbing near-vertical and vertical surfaces. The baseline wall material for the Soft-Rough testing was carpet. Carpet was also chosen as the primary development surface as it is easy to maintain good attachment, allowing behavior (maneuverability and body dynamics) to be partially decoupled from foot adhesion. The climbing foot used for carpet is a single point dactyl foot, as depicted in Figure 10 (a). For the Soft-Smooth surface a cork material was chosen. Initially we expected cork to be a simple surface as it was a soft surface easily penetrated by spines and dactyls, this however was not the case. Cork proved to be challenging due to the relative material strength vs. the mass of the robot. The Hard-Rough surface tested was hard wood planks, mounted parallel to the vertical axis of the wall, which were climbed reliably ${ }^{*}$ up to $55 \mathrm{deg}$. Wood planks proved challenging due to frequent jamming of the dactyl when placed down in the cracks between planks. Recently work has begun developing traction controlled climbing gaits for purely vertical stucco surfaces [26]. And finally the Hard-Smooth surfaces tested on are both acrylic sheets and smooth concrete board.

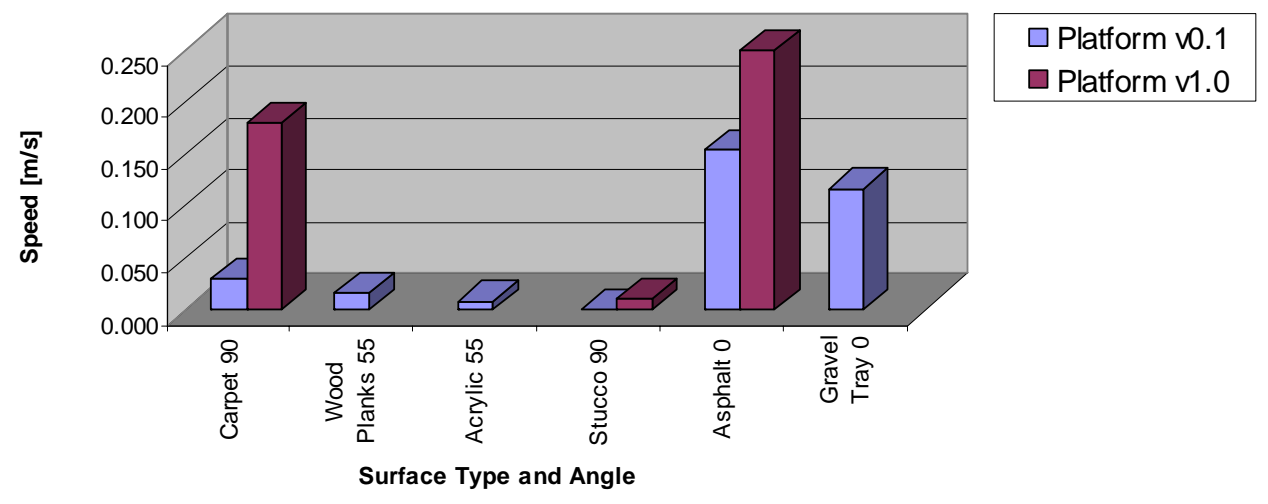

Figure 11: Robot climbing performance for a variety of test surfaces from horizontal to vertical.

Although rudimentary, some testing in the horizontal regime has been completed. For flat surfaces such as asphalt the robots current walking speed for v0.1 is currently $0.15 \mathrm{~m} / \mathrm{s}$. We expect the new compliant v1.0 leg design to achieve much higher speeds once proper values for spring constant and damping have been identified. For rougher surfaces such as the gravel bed at SwRI, where surface variations are close to $20 \%$ leg length, the v0.1 robot walks at $0.12 \mathrm{~m} / \mathrm{s}$. No official testing has been done to date to determine the maximum obstacle height the RiSE platform is capable of traversing in the horizontal regime.

Preliminary endurance tests were completed with the v0.1 platform on a vertical carpet surface. The robot was walked up and down the wall continuously until the battery voltage fell below the operational threshold. The average run time was close to 15 minutes. With one of the three batteries installed in the new v1.0 platform initial results suggest a continuous run time of $20 \mathrm{~min}$, projected runtime for a three pack configuration is 50-60 min. The payload capacity of

* Reliable climbing was determined by three successful back to back tests over a three meter distance 
the v0.1 robot is roughly $1.5 \mathrm{~kg}$ before discernable performance changes at low climbing speeds, approximately $1 / 3$ the max climbing speed. Additionally, RiSE has been tested with the dactyl feet on chain link fences, tree trunks and a brick walls.

\section{CONCLUSION}

We described the biological motivation and the design strategy for RiSE, a power autonomous six legged climber. RiSE aims to emulate the fundamentally necessary force patterns for climbing, as exhibited in biological climbers and numerical models of the platform, while striking a balance with the numerous engineering constraints facing robot designers. The result is a platform capable of climbing on a large variety of surfaces, using only two actuators per leg. We have developed a set of feet, each with a range of surface attachment abilities. In the future we plan to integrate these feet into multifunctional appendages, inspired by biological feet, to increase the versatility of RiSE. A key component of such multifunctional feet will be artificial dry adhesives currently under development by Fearing et.al. at Berkeley [28].

\section{ACKNOWLEDGEMENTS}

The RiSE platform is the result of the ingenuity, hard work and talent of a large number of people, including robotics and biology groups at Boston Dynamics, U. Pennsylvania, Carnegie Mellon, Stanford, Berkeley and Lewis\&Clark. This work is supported by a Defense Advanced Research Projects Agency within the DSO Biodynotics Program under contract DARPA/SPAWAR N66001-03-C-8045.

\section{REFERENCES}

1. L. Guo, K. Rogers, and R. Kirkham, A Climbing Robot with Continuous Motion, Proc. IEEE International Conference on Robotics and Automation, San Diego, CA, 1994.

2. S. Hirose, A. Nagabuko, and R. Toyama, Machine That Can Walk And Climb on Floors, Walls and Ceiling, Proc. International Conference on Advanced Robotics and Automation, France, 1992.

3. M. Minor, M., H. Dulimarta, G. Danghi, R. Mukherjee, R. Lal Tummala, and D. Aslam, Design, implementation, and evaluation of an under-actuated miniature biped climbing robot, Proc. IEEE/RSJ International Conference on Intelligent Robots and Systems, 2000.

4. K. Yoneda, Y. Ota, K. Hirano, and S. Hirose, Development of a Light-Weight Wall Climbing Quadruped with Reduced Degrees of Freedom, Proc. 4th International Conference on Climbing and Walking Robots, 2001.

5. J. Bares, and D. Wettergreen, Dante II: Technical Descriptions, Results and Lessons Learned, International Journal of Robotics Research, 1999.

6. B.L. Luk, D.S. Cooke, A.A. Collie, N.D. Hewer, and S. Chen, Intelligent Legged Climbing Service Robot For Remote Inspection And Maintenance In Hazardous Environments, Proc. 8th IEEE Conference on Mechatronics and Machine Vision in Practice, Hong Kong, 2001.

7. B. Kennedy, A. Okon, H. Aghazarian, M. Badescu, X. Bao, Y. Bar-Cohen, Z. Chang, B.E. Dabiri, M. Garrett, L. Mangnone, and S. Sherrit, Lemur IIIb: A Robotic System for Steep Terrain Access, Proc. 9th International Symposium on Experimental Robotics, Singapore, June, 2004.

8. K.A. Daltorio, A.D. Horchler, S. Gorb, R.E. Ritzmann, and R.D. Quinn, A Small Wall-Walking Robot with Compliant, Adhesive Feet, Proc. IEEE International Conference on Intelligent Robots and Systems, Alberta, Canada, 2005.

9. R.D. Quinn, G.M. Nelson, R.J. Bachmann, D.A. Kingsley, J. Offi, and R.E. Ritzmann, Insect Designs for Improved Robot Mobility, Proc. $4^{\text {th }}$ International Conference on Climbing and Walking Robots, Karlsruhe, Germany, 2001.

10. K.A. Daltorio, S. Gorb, A. Peressadko, A.D. Horchler, R.E. Ritzmann, and R.D. Quinn, A Robot that Climbs Walls using Micro-structured Polymer Feet, Proc. International Conference on Climbing and Walking Robots, London, UK, 2005.

11. C. Menon, M. Murphy, and M. Sitti, Gecko Inspired Surface Climbing Robots, Proc. IEEE International Conference on Robotics and Biomimetics, Shenyang, China, 2004.

12. P. Menzel and F. D’Aluisio, Robo Sapiens: Evolution of a new Species, MIT Press, 2000. 
13. A. Greenfield, A. Rizzi, and H. Choset, Dynamic Ambiguities in Frictional Rigid-body Systems with Application to Climbing via Bracing, Proc. IEEE International Conference on Robotics \& Automation, Barcelona, Spain, 2005.

14. G. Granosik, M.G. Hansen, and J. Borenstein, The OmniTread Serpentine Robot for Industrial Inspection and Surveillance, Proc. International Journal on Industrial Robots, Special Issue on Mobile Robots, vol. IR32-2, 2005.

15. Clarifying Technologies Inc., Climber III - Remote Controlled Climbing Vehicle (Gen3), www.clarifyingtech.com, USA.

16. U. Saranli, M. Buehler, and D. Koditschek, RHex: A Simple and Highly Mobile Hexapod Robot, International Journal of Robotics Research, 2001.

17. K. Autumn, Properties, principles, and parameters of the gecko adhesive system. In A. Smith and J. Callow, Biological Adhesives. Springer-Verlag, 2006.

18. A.T. Asbeck, S. Kim, W.R. Provancher, and M. Lanzetta, Scaling hard vertical surfaces with compliant microspine arrays, Proceedings of Robotics: Science and Systems, Cambridge, USA, 2005.

19. M. Spenko, M. Cutkosky, C. Majidi, R. Fearing, R. Groff, and K. Autumn, Foot design and integration for bioinspired climbing robots, Proc. SPIE Defense\&Security Symposium, Unmanned Systems Technology, Orlando, FL, 2006.

20. S.A. Bailey, J.G. Cham, M.R. Cutkosky, and R.J. Full. Biomimetic mechanisms via shape deposition manufacturing, International Symposium on Robotic Research. Springer-Verlag, London, 1999.

21. K. Autumn, S.T. Hsieh, D.M. Dudek, J. Chen, C. Chitaphan, and R.J Full, Dynamics of Geckos Running Vertically, The Journal of Experimental Biology, 2006.

22. D.I. Goldman, T.C. Chen, and R.J. Full. A Template for Rapid Vertical Climbing, Society of Integrative and Comparative Biology, Annual Meeting \& Exhibition, Orlando, FL, 2006.

23. J. Schmitt, M. Garcia, R.C. Razo, P. Holmes, and R.J. Full, Dynamics and stability of legged locomotion in the horizontal plane: A test case using insects. Bio. Cyber., 86 (5), 2002.

24. K. Autumn, M. Buehler, M. Cutkosky, R. Fearing, R.J. Full, D. Goldman, R. Groff, W. Provancher, A. Rizzi, W. Saranli, A. Saunders, and D.E. Koditschek, Robotics in Scansorial Environments, Proc. SPIE Defense\&Security Symposium, Orlando, FL, 2005.

25. Maxon Precision Motors Inc., RE Motor Series, http://www.maxonmotor.com/

26. A.A. Rizzi, C. Haynes, D.E. Koditschek, and R.J. Full, Gait generation and control in a climbing hexapod robot, Proc. SPIE Defense\&Security Symposium, Unmanned Systems Technology, Orlando, FL, 2006.

27. W.P. Provancher, J.E. Clark, B. Geisler, and M.R. Cutkosky, Towards Penetration-based Clawed Climbing, Int. Conf. on Climbing and Walking Robots, Madrid, Spain, 2004.

28. M. Sitti and R.S. Fearing, Synthetic Gecko Foot-Hair Micro/Nano-Structures for Future Wall-Climbing Robots, Proc. IEEE International Conference on Robotics and Automation, Taiwan, 2003. 